\title{
Concepção e Avaliação de Tecnologia mHealth para Promoção da Saúde Vocal
}

Daniele de Araújo Oliveira Carlos${ }^{1}$, Thiago de Oliveira Magalhães ${ }^{2}$, José Eurico Vasconcelos Filho ${ }^{2}$, Raimunda Magalhães da Silva ${ }^{1}$, Christina Cesar Praça Brasil ${ }^{1}$

danii_joly@yahoo.com.br, dev.thiago.magalhães@gmail.com, euricovasconcelos@unifor.br, rmsilva@unifor.br, cpraca@unifor.br

${ }^{1}$ Programa de Pós Graduação em Saúde Coletiva, Universidade de Fortaleza, Fortaleza, Ceará, Brasil.

${ }^{2}$ Nucleo de Aplicação em Tecnologia da Informação -NATI, Universidade de Fortaleza, Fortaleza, Ceará, Brasil.

DOI: 10.17013/risti.19.46-60

Resumo: O estudo aborda a concepção, o desenvolvimento e a avaliação de um aplicativo para dispositivos móveis que auxilie no cuidado e gerenciamento da saúde vocal. A pesquisa desenvolveu-se em três fases: 1) levantamento bibliográfico, 2) concepção e desenvolvimento do artefato tecnológico em laboratório e 3) avaliação preliminar do aplicativo por fonoaudiólogos. Para a concepção e desenvolvimento do aplicativo utilizaram-se recursos multimídia, visando à apresentação clara dos conteúdos educativos; além de funcionalidades personalizadas que apoiassem na identificação de situações de risco vocal. Durante a avaliação, fonoaudiólogos, que atuaram como juízes, utilizaram o aplicativo e emitiram seus pareceres. Os dados foram analisados com base na Análise de Conteúdo. Os resultados mostram que a ferramenta pode auxiliar os profissionais a cuidarem da sua voz e dar suporte aos fonoaudiólogos no acompanhamento dessa população, evidenciando que, na saúde coletiva, a tecnologia é capaz fortalecer as estratégias de educação e promoção da saúde.

Palavras-chave: Voz; Tecnologia; mHealth; Estudo de Avaliação; Análise de Conteúdo

Abstract: The study addresses the design, development and evaluation of an app for mobile devices that assist in the care and management of vocal health. The research was carried out in three phases: 1) literature review, 2) laboratory design and development and 3) preliminary assessment of the app by speech therapists. For the design and app development, multimedia features were applied, in order to offer clear presentation of the educational content; as well as customized resources that support the identification of vocal risk situations. During the evaluation, speech therapists, who acted as judges, handled the app and issued their opinions. Data were analyzed based on content analysis. The results show that the app can help professionals to take care of their voices and give support to the speech therapists in monitoring this population, highlighting that, in public health, technology is able to strengthen the education and health promotion strategies.

Keywords: Voice; Technology; mHealth; Evaluation Studies; Content Analysis 


\section{Introdução}

No Brasil, o uso da tecnologia na assistência à saúde ainda é um desafio. As inovações tecnológicas existentes respondem por uma parcela significativa de esforço em pesquisa. No entanto, há a necessidade de aprofundamento dos conhecimentos em saúde, bem como da implementação de iniciativas que busquem um modelo de desenvolvimento socialmente inclusivo e economicamente competitivo (Gadelha \& Costa, 2012).

As Tecnologias da Informação e Comunicação (TICs) desafiam a transformação dos conceitos educacionais, perspectivas didáticas e convidam os pesquisadores a revisarem e complementarem suas formações, levando-os a refletir sobre novas possibilidades e conhecimentos que podem ser agregados à rotina diária, facilitando e estreitando as relações (Weinert, 2013).

Ciência e tecnologia são elementos importantes para a promoção da saúde e para o tratamento das doenças, assim como para a construção de novos conhecimentos e ferramentas como centro estratégico para a sobrevivência e crescimento de instituições e organizações. Seria inimaginável a sobrevivência sem determinados recursos e instrumentos como, por exemplo, a energia elétrica, o computador, o telefone fixo ou o celular. Do mesmo modo, parece inconcebível manter e garantir a saúde sem os medicamentos, próteses, instrumentos cirúrgicos, vacinas, equipamentos para a realização de exames, etc. Muitas vezes, o homem esquece de associar o quanto a tecnologia se faz presente na saúde (Lorenzetti et al., 2012).

Nos últimos anos, a área de pesquisa em saúde móvel ( $m$ Health), ramo da saúde eletrônica (eHealth) que é definido como "o uso de tecnologias de computação e comunicações móveis em cuidados de saúde e de saúde pública”, tem estado em constante expansão. As aplicações móveis para a saúde atendem a um público heterogêneo - médicos, enfermeiros, pacientes, cuidadores ou mesmo pessoas saudáveis (Free et al., 2010) - e a uma grande variedade de fins (Riley et al., 2011), tais como: informações nas mais diversas áreas da saúde, adesão a tratamento(s) e gestão da doença.

As maiores vantagens da utilização de dispositivos móveis para a saúde consistem no fato de que estes recursos são pessoais, inteligentes (têm capacidade de processamento), são dotados de sensores, possuem conexão com a Internet e são portáteis (Whittaker, 2012). Portanto, eles podem atender aos usuários, tanto na vida cotidiana quanto durante a internação ou reabilitação. Também podem atender aos prestadores de cuidados à saúde durante as visitas de rotina ou de emergência.

O contexto atual sugere que o uso da tecnologia móvel pode apoiar o diagnóstico e o cumprimento das diretrizes de tratamento, bem como guardar informações sobre os pacientes, além de aumentar a eficiência administrativa (Sherry \& Ratzan, 2012, Vázquez et al., 2016). Com isso, concebeu-se uma abordagem tecnológica mHealth que permita a professores e profissionais que usam intensivamente a voz conhecer mais sobre saúde vocal e seu cuidado, além de dispor de recursos para monitorar a sua própria voz (inclusive em tempo real), de forma integrada ao seu ambiente de trabalho e dinâmica cotidiana. 
Diante do exposto, a presente pesquisa justifica-se pelo grande número de trabalhos que abordam a saúde vocal do professor, no entanto, o número de professores afastados por problemas relacionados à voz só aumenta no cenário brasileiro, fazendose necessário criar estratégias inovadoras que possam ser empregadas para promover o autocuidado e minimizar as alterações vocais desses profissionais. Com base nessas reflexões afloraram as seguintes questões: O que os profissionais da voz conhecem sobre a voz?, Como os profissionais da voz cuidam da saúde vocal?, Que fatores de risco estão presentes na prática dos profissionais da voz que podem comprometer a saúde vocal?, Quais impactos as alterações vocais trazem para a vida pessoal e profissional desses profissionais?, Que ações são desenvolvidas para beneficiar a saúde vocal desses profissionais?, Que ferramentas são disponibilizadas para que os professores monitorem e cuidem da sua voz? E Será que os professores têm amplo acesso a serviços de saúde para o cuidado com a voz?

Realizou-se um levantamento bibliográfico sobre a os recursos tecnológicos existentes no mercado e academia para o gerenciamento e o cuidado com a saúde vocal, dentre estes, foram identificados quatro que se destacam, tendo em vista os objetivos propostos, quais sejam: Vocal WarmUp, Saúde Vocal, Vocal Ease, Warm Me Up for Singers e VoxMetria. Observou-se, entretanto, que as ferramentas identificadas têm como principal característica o fornecimento de informações, sem interfaces interativas que promovam o monitoramento da função vocal e o arquivamento de registros necessários ao gerenciamento do uso da voz e das condições ambientais.

Diante dos resultados do levantamento, observa-se que não há recursos disponíveis para o pleno gerenciamento e o cuidado com a saúde vocal dos professores que atendam aos questionamentos identificados. Entende-se que estes são questionamentos importantes e que podem ser respondidos com o desenvolvimento desta pesquisa.

Assim, acredita-se que a elaboração de um aplicativo para o monitoramento, autogerenciamento e autocuidado com a saúde vocal constitui uma resposta aos questionamentos levantados em estudo anterior (Brasil, 2015), o que poderá favorecer a redução dos acometimentos e a recorrência das alterações vocais (Carlos et al., 2016).

Diante do exposto, o presente estudo tem como objetivo desenvolver e avaliar preliminarmente um aplicativo para dispositivos móveis que auxilie profissionais da voz no gerenciamento da saúde vocal.

\section{Metodologia}

Trata-se de um estudo qualitativo, uma vez que resultados traduzidos em números não serão suficientes para expressar as experiências dos sujeitos da pesquisa (Costa, Reis \& Souza, 2014). É de natureza exploratória, experimental e aplicada, realizada em Fortaleza, Ceará - Brasil, de novembro de 2014 a dezembro de 2015.

A execução do projeto aconteceu em três etapas (Carlos et al., 2016). A primeira fase consistiu em uma revisão da literatura sobre a temática e o contexto proposto, permitindo a equipe de pesquisa uma melhor apropriação da problemática envolvida e de conceitos, metodologias e ferramentas das áreas envolvidas. A segunda fase, laboratorial, teve 
como objetivo a concepção e o desenvolvimento do artefato tecnológico, o aplicativo. $\mathrm{Na}$ terceira fase, foi realizada uma avaliação preliminar do aplicativo por profissionais especialistas em voz (fonoaudiólogos).

A fase de concepção e desenvolvimento do aplicativo teve início em novembro de 2014 e foi realizada no laboratório de Inovação Tecnológica da Universidade de Fortaleza UNIFOR, com o auxílio de uma equipe multidisciplinar. Para o desenvolvimento foi adotado um processo próprio da área de Interação Humano Computador, o design de interação participativo (Preece, Rogers \& Sharp, 2013), escolhido por ser centrado nas necessidades do usuário e contar com a participação contínua deste no processo. O Design de Interação subdivide-se em quatro atividades: identificar necessidades (dos usuários) e estabelecer requisitos (para o sistema); projetar o design da solução e, quando necessário o redesign, construir uma versão interativa (protótipo funcional) e avaliar a versão produzida junto aos usuários.

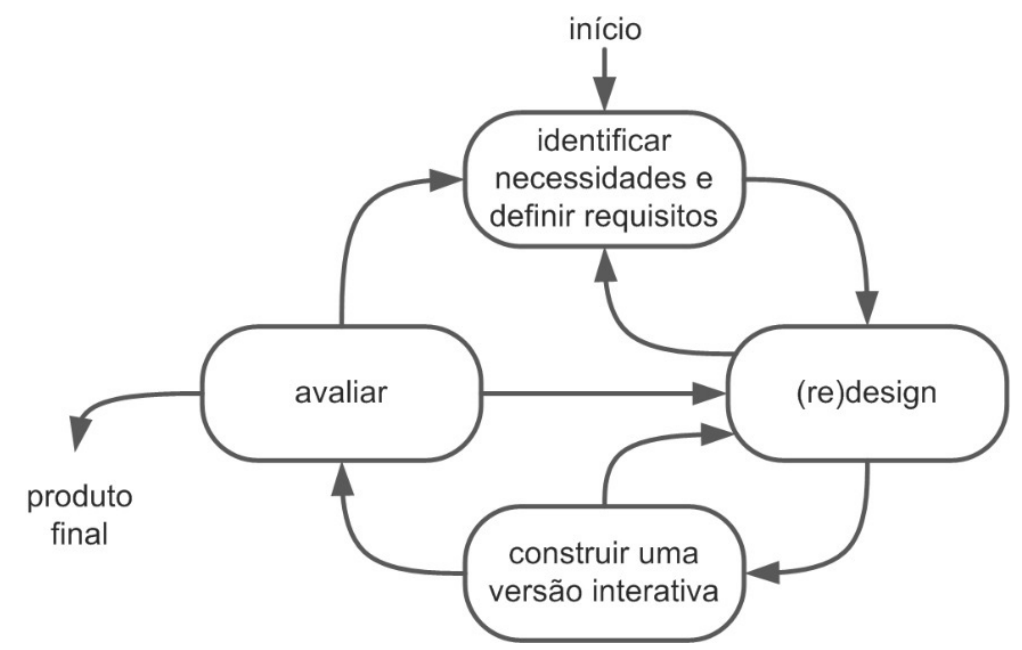

Figura 1 - Design de interação participativo (Preece, Rogers \& Sharp, 2013)

Como resultado da primeira atividade do processo de design (identificar necessidades e estabelecer requisitos), identificaram-se alguns requisitos para o artefato a ser codificado, dentre eles:

- Cadastro de usuário e login: definição do usuário no sistema para registro e integração identificada aos serviços na web.

- Definição do Perfil: Por meio dessa funcionalidade o usuário responderá a algumas questões e, a partir dos resultados, obterá uma classificação sobre o nível de proteção vocal.

- Compartilhamento de dados nas redes sociais: O usuário terá a opção de compartilhar seus resultados ou informações nas redes sociais.

- Análise de predisposição a problemas vocais: Por meio de cinco questões norteadoras, o usuário será classificado dentro de uma escala que varia de o a 5 que representa a tendência de desenvolver um problema vocal. 
- Escudos de proteção vocal: Trata-se de uma ferramenta de gamificação (Chou, 2015) que, de acordo com o uso do aplicativo e ações do usuário, o usuário terá, em uma escala de o a 5, o nível atual de proteção da sua voz contra problemas vocais.

- Dicas de saúde vocal: Orientações sobre o que faz bem e o que faz mal a voz.

- Visão principal do aplicativo: Local onde serão mostrados os principais ambientes do aplicativo.

- Análise vocal: Recurso que possibilita o professor gravar a sua voz e, por meio desse material, o software calcula o Tempo Máximo de Fonação (TMF), utilizando a relação $\mathrm{S} / \mathrm{Z}$, e informa ao usuário como está o funcionamento das pregas vocais.

- Visualização de Resultados: Essa função permitirá ao usuário visualizar o resultado obtido a partir da análise vocal (TMF), seguido de um texto explicativo.

- Histórico de uso: Neste ambiente, o usuário poderá visualizar os gráficos de uso e evolução com o uso do aplicativo. Além disso, nessa funcionalidade ficarão memorizadas as gravações de voz e as alterações vocais registradas durante todo o uso do aplicativo.

Uma vez estabelecidos os requisitos e, com base neles, iniciou-se a atividade de design, na qual são construídos os desenhos das interfaces do sistema (telas) de modo a contemplar os requisitos, além de usabilidade e estética. Esses desenhos são compreensíveis para toda a equipe, permitindo revisões com a participação de potenciais usuários da proposta.
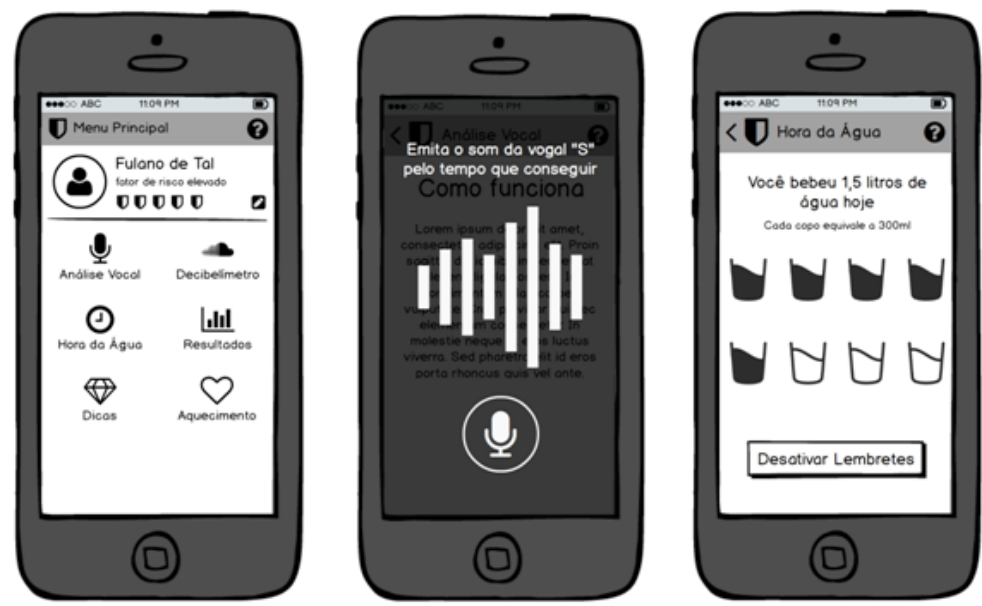

Figura 2 - Desenhos das interfaces. As telas representam respectivamente o Menu Principal, a tela de Análise Vocal e a tela de acompanhamento da funcionalidade Hora da Água.

Finalizadas as revisões do artefato (design e redesign), deu-se início à fase de construção da versão interativa. Nessa etapa, é onde ocorre à programação em si, a codificação do artefato tecnológico (Cabrera et al., 2015). Para essa atividade, a equipe utilizou as melhores práticas e ferramentas das áreas de Interação Humano Computador e Engenharia de Software. A plataforma escolhida para o aplicativo foi a Android, tendo 
em vista dominar mais de 80\% do mercado brasileiro de dispositivos móveis. Como resultado da atividade, gerou-se a primeira versão do VoiceGuard sendo algumas das telas apresentadas a seguir:

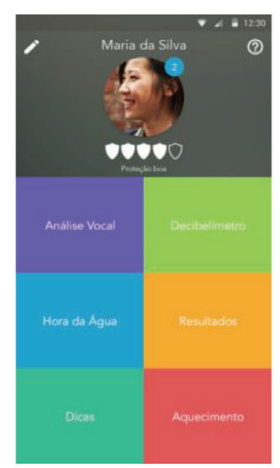

Menu principal do aplicativo

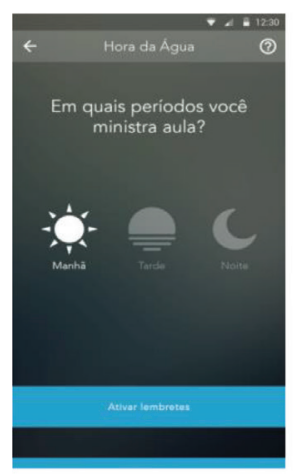

Tela hora da água

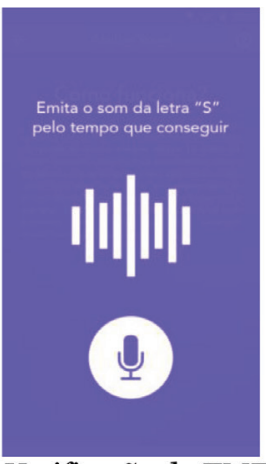

Verificação do TMF

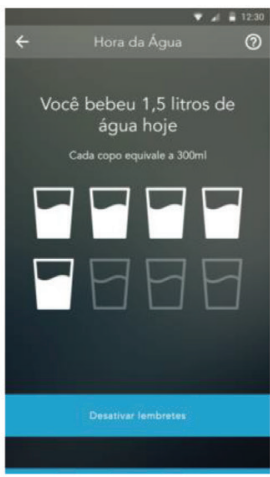

O usuário registra o consumo de água
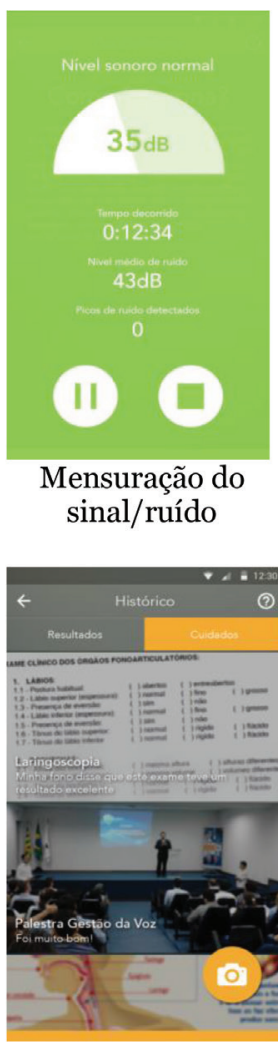

Tela para registro do histórico

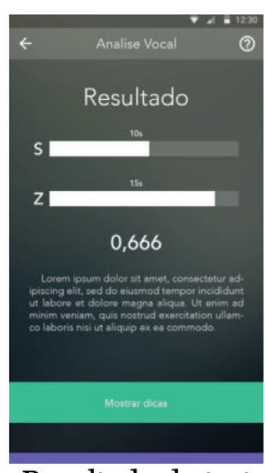

Resultado do teste realizado (TMF)

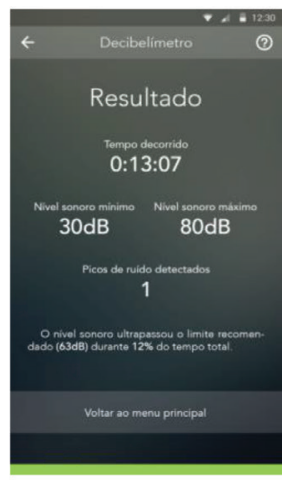

Resultados obtidos da mensuração do ruído

Figura 3 - Interfaces do protótipo de alta fidelidade. Apresentação de oito das 16 telas do aplicativo

Aúltima atividade doprocesso correspondeu a avaliaçãodeusabilidadedoaplicativo(Barbosa \& Silva, 2010). Essa avaliação tem como objetivo verificar se o aplicativo é compreensível, fácil de usar e gera uma boa experiência para seus usuários. Essa etapa, entretanto, não avalia o conteúdo ou se o artefato produzido responde aos objetivos propostos, ficando essa avaliação para a fase seguinte da metodologia. Como resultado da avaliação de usabilidade, obteve-se indícios positivos de que os usuários se apropriaram com facilidade do aplicativo, conseguindo utilizá-lo da forma adequada e tendo uma boa experiência de uso.

A terceira fase da metodologia contemplou a avaliação do aplicativo desenvolvido por especialistas em voz, da qual participaram três fonoaudiólogos que fizeram a análise do 
aplicativo no que diz respeito aos seguintes aspectos: estrutura geral (composta pelo layout e o conteúdo das telas), facilidade de uso e atendimento aos objetivos propostos.

O processo de avaliação, que corresponde à coleta de dados, iniciou-se com uma reunião explicativa para que os profissionais entendessem o contexto e o objetivo da pesquisa. Esta reunião foi conduzida por duas pesquisadoras. Em seguida, o aplicativo, já instalado em celulares com tecnologia Android, foi disponibilizado para a experimentação e avaliação dos profissionais por quatro horas. Decorrido esse tempo, foi aplicado um guia de avaliação e cada profissional fez apontamentos sobre o protótipo do VoiceGuard, emitindo suas opiniões de forma oral e escrita. Ressalta-se que os relatos orais foram obtidos por meio de uma entrevista semiestruturada realizada pelas pesquisadoras com os fonoaudiólogosavaliadores, tendo sido gravadas com gravador digital. As opiniões escritas foram registradas no guia de avaliação entregue aos fonoaudiólogos. Todos os profissionais assinaram o Termo de Consentimento e Livre Esclarecimento, concordando com a participação no estudo.

Os dados foram transcritos com base nas gravações. Para proteger a identidade dos participantes, os mesmos foram denominados por letras e números (1 a 3). Assim, a letra "F" significa fonoaudiólogo, conforme o exemplo: F1 remete ao fonoaudiólogo 1.

Em seguida, os dados foram tratados com base na Análise de Conteúdo na modalidade temática (Bardin, 2011), evidenciando-se duas temáticas de análise, quais sejam: estrutura visual e aplicação e eficácia do aplicativo. Esta técnica corresponde a um conjunto de estratégias que possibilitam a análise de diversos tipos de comunicação,

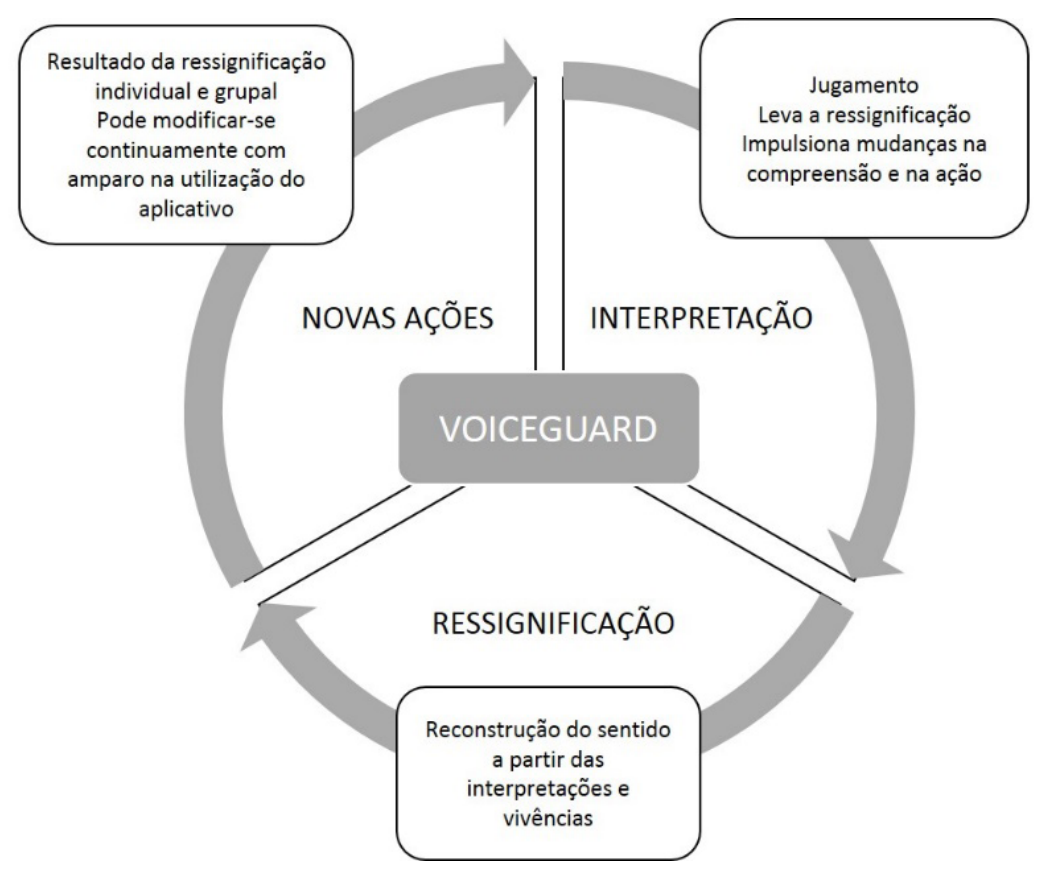

Figura 4 - Representação cíclica do Interacionismo Simbólico 
com o objetivo de obter um maior conhecimento sobre o assunto em pauta dentro de um contexto mais específico.

A interpretação dos dados foi realizada com base no Interacionismo Simbólico (Blumer, 1969) e nas teorias sobre as aplicações da tecnologia na saúde e mHealth. Nesse percurso metodológico, verificou-se o caráter cíclico do Interacionismo Simbólico, o que já havia sido apontado por Brasil (2015) e Brasil \& Silva (2016). No caso do presente estudo, interligam-se interpretações, ressignificações e novas ações. Assim, verifica-se que a perspectiva interacionista possibilita, por meio da interação social que ocorre entre uma tecnologia mHealth e a mediação de um profissional da saúde, a interpretação e a ressignificação de contextos, resultando nas mudanças de condutas e atitudes.

Os procedimentos ético-legais da pesquisa seguiram as normas contidas na Resolução $\mathrm{n}^{\mathrm{O}}$ 466, de 12 de dezembro de 2012, do Conselho Nacional de Saúde, a qual aponta as Diretrizes e Normas Regulamentadoras de Pesquisa Envolvendo Seres Humanos (Brasil, 2012). A pesquisa está vinculada como desdobramento da tese de doutorado intitulada "A voz da professora não pode calar: sentidos, ações e interpretações no contexto da integralidade em saúde”, a qual teve aprovação pelo Comitê de Ética sob o n ${ }^{\circ}$ 99.798.

\section{Resultados e discussão}

Como resultado da primeira etapa, obteve-se um protótipo de alta fidelidade com 16 telas e as seguintes funções:

- $\quad$ perfil - nesta tela, o usuário responde a alguns questionamentos e, a partir dos resultados obtidos com as respostas, obtém uma classificação sobre o nível de proteção vocal;

- compartilhamento de dados - o usuário pode compartilhar seus resultados nas redes sociais;

- análise de predisposição a problemas vocais - o usuário é classificado dentro de uma escala que representa a tendência que ele tem de desenvolver um problema vocal;

- nível de proteção vocal - ferramenta de gamificação que, de acordo com o uso do aplicativo, o usuário tem, em uma escala de o a 5, o nível atual de proteção da sua voz contra problemas vocais;

- dicas sobre saúde vocal -orientações sobre o que faz bem e o que faz mal à voz;

- análise vocal - recurso que possibilita a gravação da voz pelousuário e a obtenção do cálculo do Tempo Máximo de Fonação (TMF), utilizando a relação $\mathrm{S} / \mathrm{Z}$, e informa ao usuário como está o funcionamento das pregas vocais;

- resultados - nesta tela, o indivíduo tem a oportunidade de visualizar o resultado obtido a partir da análise vocal (TMF), seguido de um texto explicativo;

- histórico de uso - neste ambiente, o professor pode visualizar os gráficos de uso e evolução com o uso do aplicativo;

- decibelímetro - ferramenta capaz de mensurar o nível de ruído ambiental;

- hora da água - ferramenta que programa e lembra o usuário de realizar a ingestão de água para o bom uso da voz;

- aquecimento e desaquecimento vocais - mostra exercícios vocais para o usuários. 
Na segunda etapa, a partir das entrevistas com os fonoaudiólogos para a avaliação preliminar do aplicativo, identificaram-se duas temáticas, quais sejam: "estrutura visual” e "aplicação e eficácia”.

\subsection{Estrutura visual}

A estrutura visual do aplicativo foi um aspecto muito evidenciado pelos profissionais que o avaliaram, uma vez que o layout e os conteúdos das telas funcionam como porta de entrada para que o usuário se interesse pelo produto, conforme o seguinte relato: "As telas são muito bonitas e as cores chamam a atenção...” (F3); “...O conteúdo está bacana, o visual também é bacana para a pessoa ver, para ela querer usar..., agora, tem uma certa dificuldade de avaliar inicialmente, mas eu acho que é bacana...”(F2).

Um estudo realizado por Galvão \& Püschel (2012), que teve como proposta a construção e a avaliação de um aplicativo para dispositivo móvel verificou que a imagem visual, além de fomentar a motivação, promove o conhecimento intuitivo e possibilita a compreensão de conceitos que, se fossem dispostos somente em forma de texto, seriam mais difíceis de serem absorvidos ou memorizados.

As vantagens de um aplicativo que possui a associação de hipertexto, imagem e interação com o usuário promovem rapidez do processo de aprendizagem, oferta de ambientes para os profissionais explorarem, possibilitando o resgate de informações relevantes no ato de recordar, o que por sua vez, facilita a promoção da saúde vocal. Além disso, favorece a construção do conhecimento, permitindo ao usuário definir o seu próprio caminho, o que traduz o potencial para a educação em saúde.

Outro estudo realizado por Quiñonez, Luzardo \& Granda (2016) que teve como objetivo implementar um sistema multi-touch em ambientes educacionais para promover e facilitar a avaliação do trabalho colaborativo em sala de aula evidenciou que o uso datecnologia tem um impacto positivo sobre a percepção dos professores e alunos. Os professores relataram que o sistema facilita a avaliação do trabalho colaborativo feito por estudantes. Por outro lado, os alunos relatam que essas ferramentas ajudam a refletir mais sobre o conteúdo ministrado em sala de aula, além de facilitar o processos de ensino e aprendizagem.

No que diz respeito ao conteúdo das telas, todos os profissionais concluíram que itens como análise vocal, aquecimento e desaquecimento não seriam suficientes para responder à proposta inicial do aplicativo, o que ficou evidente na seguinte fala: "Eu só achei que essa análise vocal é pouca...eu acho o teste da relação $S / Z$ muito pouco... Como é que você vai evoluir alguém só com a relação $S / Z$, minha gente?” (F1). Outro relato que reforça a avaliação desses aspectos foi o seguinte: "Esse negócio de aquecimento $e$ desaquecimento é muito particular, é individual... além disso, eu evoluo meus pacientes todas as semanas com aquecimento e desaquecimento" (F2).

A medida do Tempo Máximo de Fonação (TMF) é um teste muito utilizado para se verificar a qualidade vocal, utilizando, dentre outros, os fonemas fricativos /s/ e /z/ (Behlau et al., 2001). Ao emitir um determinado fonema de forma sustentada, após uma única expiração, cronometra-se esse tempo, o qual fornece dados sobre a dinâmica da vocalização, sendo bastante fidedigno na avaliação da eficiência glótica. Ao finalizar a emissão de um fonema, outro pode ser requerido. A utilização dos fonemas /s/ e /z/, 
permite estabelecer uma relação, a qual possibilita verificar a condição da coaptação glótica. Ressalta-se que o padrão de normalidade de uma relação s/z é estimado em aproximadamente 1 (Gelfer \& Pazera, 2006).

Apesar da eficácia deste teste, os fonoaudiólogos que avaliaram o aplicativo consideraram a necessidade de explicações preliminares para os usuários o realizarem de forma autônoma; com a ressalva de uma possibilidade de distorções na realização do teste isoladamente ou na mensuração indevida do risco vocal. Diante disso, sugeriram alterar ou acrescentar ao VoiceGuard outros recursos para favorecer uma maior eficácia para esse tipo de monitoramento vocal.

As técnicas de aquecimento e desaquecimento vocais auxiliam os profissionais da voz a obterem um melhor desempenho no exercício profissional (Aydos \& Hanayama, 2004). Entretanto, essas técnicas precisam ser apresentadas aos usuários por especialistas para que sejam corretamente realizadas. Assim, ao utilizar o aplicativo VoiceGuard, os fonoaudiólogos verificaram a necessidade de uma apresentação mais real e ilustrada, de preferência, em forma de imagens dinâmicas para evitar que os usuários do aplicativo realizem inadequadamente os exercícios. Ademais, o contato inicial dos fonoaudiólogos com o profissional da voz isoladamente ou em grupo foi considerado de elevada importância para instrumentalizar o uso do aplicativo.

Com base no que exposto, as telas que, inicialmente, correspondiam à análise, ao aquecimento e desaquecimento vocais, estão no laboratório para serem substituídas por novas telas, com funções aprimoradas, de forma a suprir as fragilidades identificadas na estrutura do aplicativo.

\subsection{Aplicação e a eficácia do aplicativo}

A partir dos relatos dos avaliadores, foi possível perceber que o dispositivo deveria ser direcionado para os fonoaudiólogos, uma vez que funciona como ferramenta de apoio à sua atuação junto aos pacientes, como demonstra a fala a seguir:

“Esse aplicativo é para a gente usar... Para a gente trabalhar com ele [o paciente], entendeu?... Eu acho super legal, por exemplo, todos os meus pacientes eu evoluo semanalmente..., os meus pacientes de voz eles só vão para mim uma vez por semana, a não ser em casos de emergência... aí, ele vem duas vezes para tratamento, mas eu prescrevo todos os exercícios e eu evoluo toda semana... Então, um aplicativo como esse para fazer e acompanhar a evolução dos pacientes seria fantástico para mim enquanto profissional... Eu acho que esse aplicativo é também muito útil para o fonoaudiólogo... talvez até mais do que para o paciente" (F1).

O relato mostra o quanto o aplicativo é considerado importante para o avaliador, como fonoaudiólogo, uma vez que visualiza muitas possibilidades para aprimorar a evolução e o monitoramento dos pacientes. Diante dessa opinião, a pesquisadora explicitou que essa ferramenta, para ser bem utilizada pelos profissionais da voz (professores, cantores, outros), precisa da interveniência do fonoaudiólogo, uma vez que o manuseio correto precisa ser apresentado numa sessão inicial - treinamento para a promoção da saúde vocal com amparo no VoiceGuard; após o que os usuários terão autonomia para utilizar a ferramenta. Assim, o aplicativo constitui uma ferramenta a mais para instrumentalizar 
fonoaudiólogos nas ações de promoção da saúde vocal, podendo também ser aplicada ao seguimento de pacientes em terapia.

Cada vez mais, a tecnologia funciona como aliada aos profissionais da saúde, o que pode ser constatado pelos resultados de um estudo de Almeida \& Mello (2004), tendo evidenciado que o uso intenso da tecnologia é uma tendência de profissionais da saúde na Bahia, Brasil, revelando o impacto organizacional significativo e crescente no setor de saúde nos anos vindouros.

Outro aspecto evidenciado por Abbott \& Barbosa (2015) refere-se ao poder da combinação das tecnologias de informação e comunicação (TIC) com intervenções de mobilização social, as quais configuram como armas no enfrentamento de doenças. As autoras relatam que mesmo em países com poucos profissionais da saúde, mas com abundante telefonia celular, é possível transmitir a mensagem para grande parcela da população, por meio de TIC, mHealth e redes sociais; além disso, defendem que o uso de tecnologias de informação na saúde é muito importante para o monitoramento, vigilância de doenças e gestão da cadeia de abastecimento, o que é relevante para a saúde do planeta. Assim, deve-se incentivar que profissionais de saúde e seus pacientes utilizem mais amplamente esses recursos em sua prática e vida diária.

Os avaliadores também observaram que algumas funções do VoiceGuard precisam ser aprimoradas para garantir a maior eficácia do aplicativo e a captação mais fidedigna de informações sobre a saúde vocal, o que poderá facilitar as interpretações dos fonoaudiólogos, ao acompanhar seus pacientes, e dos usuários no autogerenciamento da saúde vocal, como mostram os relatos a seguir:

"Sabe uma coisa bacana...? O aplicativo poderia requerer a gravação da voz diariamente... É possível que o aplicativo tenha um recurso para a mensuração mais fidedigna de ruído de voz? Uma espectrografia, por exemplo. Porque... com a espectrografia você conseguiria acompanhar melhor o paciente e ele mesmo também pode acompanhar a sua qualidade vocal... porque fica documentado, entendeu?”. (F1)

“...eu acho que um aplicativo como esse tem como foco principal despertar para o problema e não resolver o problema. ...ele [o aplicativo] poderia ter uma ferramenta com um sinal de alerta para que o usuário possa acessar o fonoaudiólogo em tempo real, caso ele não consiga fazer o exercício ou o teste adequadamente... Não sei se dá para fazer assim.” (F3)

“...é interessante fazer um link das dicas oferecidas como orientações com os testes que o aplicativo realiza, por exemplo, se o usuário vai responder um questionário sobre sinais e sintomas vocais, as dicas poderiam ser direcionadas aos testes, entendeu?" (F2)

Um estudo de Souza et al. (2013) sobre a criação do aplicativo “OdontoPNE”, destinado ao cuidado odontológico de pacientes com necessidades especiais, atesta a importância das sugestões de avaliadores e de outros atores, uma vez que o recurso criado por eles dispõe de um protótipo funcional; porém reforçam que, antes da ampla disponibilização para uso por meio das lojas de aplicativos, serão requeridas melhorias na interface de usuário e otimizações de desempenho. 
Collares (2014) realizou um estudo que teve como objetivo analisar as etapas do desenvolvimento e aplicação de uma tecnologia para gestantes e profissionais da saúde. O teste de pré-validação do aplicativo foi realizado com quinze gestantes em diversos períodos gestacionais (primeiro, segundo e terceiro trimestres) e cinco profissionais de saúde (um médico e quatro enfermeiros) da Unidade de Atenção Primária à Saúde (UAPS). Apesar de ter sido muito bem avaliado pela população que compôs o estudo, foi reconhecida a necessidade do produto tecnológico ser submetido à extensiva validação para ser transferido para os serviços de saúde.

Outro estudo de Arrais \& Crotti (2015) também reforça a importância desse processo de avalição e refere-se à rápida evolução da utilização de aplicativos para a área de saúde, focando no Diabetes Mellitus. Os autores, ao conduzirem uma revisão de literatura verificaram que, dentre os 20 artigos selecionados,

“dois foram revisões sistemáticas e o maior número de artigos foram de análise crítica (seis artigos), seguidos por três artigos com análise de intervenções utilizando recursos de mHealth, e depois artigos com comentários e/ou editoriais (dois artigos), descrição de protocolos para ensaios randomizados e adaptação transcultural de instrumentos (dois artigos), e análises qualitativas de programas (dois artigos), além de um estudo randomizado e controlado de intervenção, um artigo de desenvolvimento de aplicativo e outro de descrição regulatória.” (Arrais \& Crotti, 2015, p. 129)

Diante do exposto e da importância dos processos de avaliação para favorecer a usabilidade do aplicativo, observou-se que o nível de satisfação com o VoiceGuard foi elevado, houve muitos elogios às funções "hora da água" e "decibelímetro" (para o controle do ruído ambiental). Os especialistas acreditam, entretanto, que alguns ajustes são necessários para ampliar a eficácia dos recursos e aumentar a fidedignidade das informações para os usuários, como se pode constatar nos relatos apresentados nesta temática. Os ajustes sugeridos pelos profissionais serão implementados e motivarão novas pesquisas futuras.

\section{Conclusão}

O estudo mostra que o aplicativo VoiceGuard pode auxiliar os profissionais a cuidarem da voz e dar suporte aos fonoaudiólogos no acompanhamento dessa população, evidenciando que, na saúde coletiva, a tecnologia fortalece as estratégias de educação e promoção da saúde.

Nesse sentido, a ferramenta inova o cenário da promoção da saúde vocal, uma vez que veio suprir uma necessidade evidente dos professores e de outros profissionais, no que concerne ao cuidado com a voz. Para isso, o aplicativo oferece recursos que propiciam o monitoramento e o gerenciamento da saúde vocal em tempo real.

Observa-se o reconhecimento do importante papel do fonoaudiólogo na orientação aos profissionais da voz quanto ao uso do aplicativo, especialmente no primeiro contato do usuário com a ferramenta. Neste momento, a interveniência do fonoaudiólogo otimiza o manuseio, facilita a compreensão das telas e das suas funções, além de auxiliar na compreensão dos indivíduos sobre os aspectos que envolvem a saúde vocal, favorecendo o desenvolvimento de comportamentos saudáveis. Ressalta-se que o usuário estará 
apto ao manuseio adequado da ferramenta após as devidas orientações, pelo fato de o VoiceGuard oferecer telas interativas e explicativas.

As temáticas “estrutura visual” (layout e conteúdos) e "aplicação e eficácia” do aplicativo sintetizam os principais aspectos apontados pelos fonoaudiólogos nas suas avaliações, para os quais se indentifiou a necessidade de adequações. A satisfação com o layout foi evidenciada, o que inclui imagens, cores, textos e interatividade. No que concerne ao conteúdo, apesar de ter sido considerado excelente, foram sugeridas melhorias referentes à análise vocal, ao aquecimento e ao desaquecimento vocais. Outrossim, apesar de o aplicativo ter sido considerado de fácil manuseio e de elevada eficácia no gerenciamento da saúde vocal, apontou-se a necessidade de aprimoramento de algumas funções, bem como a inclusão de novos testes.

A pesquisa será continuada a partir da implementação dos ajustes das funções propostos pelos fonoaudiólogos, no redesign de algumas telas, no redimensionamento dos parâmetros numéricos dos testes (TMF, relação $\mathrm{S} / \mathrm{Z}$, captação do nível de ruído ambiental), além de adequações no conteúdo. Em seguida, será realizado o teste de usabilidade, durante seis meses, com os professores da rede Municipal de Ensino de Fortaleza. Esses aspectos reforçam a atenção dada ao assunto pelos pesquisadores e a importância para a continuação deste estudo, o que reforça a avaliação da eficácia do VoiceGuard e da sua aplicabilidade na rotina diária dos profissionais da voz, o que será inicialmente realizado com um grupo de professores.

Esta pesquisa inspira outras propostas e ideias para grupos de estudo sobre a concepção de novas tecnologias para a área da saúde. Espera-se, ainda, que o aplicativo desenvolvido estimule os pesquisadores para que possam elaborar ferramentas que auxiliem no processo de promoção da saúde e prevenção dos agravos, nos diversos segmentos da área da saúde.

\section{Referências}

Abbott, P. A., \& Barbosa, S. F.F. (2015). Usando Tecnologia da Informação e Mobilização Social para Combater Doenças. Acta Paulista de Enfermagem, 28(1), 1.

Arrais, R.F., \& Crotti, P.L.R. (2015). Revisão: aplicativos para dispositivos móveis (“Apps”) na automonitorização em pacientes diabéticos. J. Health Inform, 7(4):127-33.

Aydos, B., \& Hanayama, E.M. (2004). Técnicas de aquecimento vocal utilizadas por professores de teatro. Rev CEFAC, 6(1), 83-88.

Barbosa, S.D.J., \& Silva, B.S. Interação Humano-Computador. Série SBC, Editora Campus-Elsevier, 2010.

Bardin, L. (2011). Análise de conteúdo. Lisboa: Edições 70.

Behlau, M., Madazio, G., Feijó, D., \& Pontes P. Avaliação de voz. (2001). In: Behlau M. organizador. Voz: o livro do especialista. vol 1. Rio de Janeiro: Revinter; 85-172.

Blumer, H. (1969) Symbolic Interactionism, Perspective and Method. California: Pretice-Hall, Inc. Englewood Cliffs, New Jersey. 208p.

Brasil (2012). Ministério da Saúde. Política Nacional de Atenção Básica. 1.ed. Brasília: Ministério da Saúde, 114p. 
Brasil, C.C.P.(2015).Avoz da professora nãopode calar: sentidos, ações e interpretações no contexto da integralidade em saúde. Tese (Doutorado em Saúde Coletiva) Universidade Estadual do Ceará, Universidade Federal do Ceará e Universidade de Fortaleza - Associação Ampla, Fortaleza.

Brasil, C.C.P., \& Silva, R.M. (2016). A voz da professora na integralidade em saúde. Fortaleza: Edições UFC.

Cabrera, A., Carrillo, J., Abad, M., Jaramillo, D., \& Romero, F. (2015). Diseño y Validación de Arquitecturas de Aplicaciones Empresariales. RISTI - Revista Ibérica de Sistemas e Tecnologias de Informação, (E4), 79-91.

Carlos, D.A. O., Magalhães, T.O., Vasconcelos Filho, J.E., Silva, R.M., \& Brasil, C.C.P. (2016). Tecnologia mHealth como Ferramenta de Promoção da Saúde Vocal. Atas CIAIQ2016 - Investigação Qualitativa em Saúde/Investigación Cualitativa en Salud. Volume 2. ISBN: 978-972-8914-59-2

Chou, Y-K. (2015). Actionable Gamification. Beyond points, badges and leaderboards. Octalysis Media.

Collares, P. M. C. (2014). Inovação tecnológica da assistência pré-natal. Tese (Doutorado em Saúde Coletiva) - Universidade Estadual do Ceará, Universidade Federal do Ceará e Universidade de Fortaleza - Associação Ampla, Fortaleza.

Costa, A. P., Reis, L. P., \& Souza, F. N. de. (2014). Investigação Qualitativa para Sistemas e Tecnologias de Informação. RISTI - Revista Ibérica de Sistemas e Tecnologias de Informação, (E2), ix-xii.

Free, C., Phillips, G., Felix, L., Galli, L., Patel V., \& Edwards P. (2010). The effectiveness of M-health technologies for improving health and health services: a systematic review protocol. BMC Res Notes, 5(14), 2-7.

Gadelha, C. A. G., \& Costa, L. S. (2012). Saúde e desenvolvimento no Brasil: avanços e desafios. Rev. Saúde Pública, São Paulo, 1(46), 13-20.

Galvão, E. C. F., \& Püschel, V. A. A. (2012). Aplicativo multimídia em plataforma móvel para o ensino da mensuração da pressão venosa central. Revista da Escola de Enfermagem da USP, 46(spe), 107-115.

Gelfer, M. P., \& Pazera, J. F. (2006). Maximum duration of sustained /s/ and /z/ and the S/z ratio with controlled intensity. $J$ Voice, 20(3), 369-379.

Lorenzetti, J., Trindade, L.L., Pires, D.E.P. de, \& Ramos, F.R.S. (2012). Tecnologia, inovação tecnológica e saúde: uma reflexão necessária. Texto contexto enferm. [online], Florianópolis, 2(21), 432-439.

Preece, J., Rogers, Y., \& Sharp, H. (2013). Design de Interação: Além da Interação Homem-Computador. 3 ed. Porto Alegre: Bookman.

Quiñonez, Y., Luzardo, G., \& Granda R. (2016). Implementación de un sistema multitáctilen ambientes educativos para promover y facilitar laevaluacióndeltrabajo colaborativo enel aula. RISTI - Revista Ibérica de Sistemas e Tecnologias de Informação, (17), 66-79. 
Riley, W.T., Rivera, D.E., Atienza, A.A., Nilsen, W., Allison, S.M., \& Mermelstein, R. (2011) Health behavior models in the age of mobile interventions: are our theories up to the task? TranslBehav Med, 1(1), 5371.

Sherry J.M., \& Ratzan S.C. (2012). Measurement and evaluation outcomes for mHealth communication: don't we have an app for that?J Health Commun, Washington, 1(17), 1-3.

Souza, R. C., Alves, L. A. C., Haddad, A. E., Macedo, M. C. S., \& Ciamponi, A. L. (2013). Processo de criação de um aplicativo móvel na área de odontologia para pacientes com necessidades especiais. Revista da ABENO, 13(2), 58-61.

Vázquez, M. Y. G., Sexto, C. F., Rocha, Á., \& Aguilera, A. (2016). Mobile Phones and Psychosocial Therapies with Vulnerable People: a First State of the Art. Journal of Medical Systems, 40(6), 1-12.

Weinert, M. E. (2013). O uso das tecnologias de informação e comunicação como ferramentas no ensino de ciências: uma proposta de trabalho interdisciplinar nos anos iniciais do ensino fundamental. Dissertação (Mestrado em Ensino de Ciência e Tecnologia) - Universidade Tecnológica Federal do Paraná, Ponta Grossa.

Whittaker, R. (2012). Issues in mHealth: findings from key informant interviews. $J$ Med Internet Res,Pittsburgh, 5(14), 129. 